Address for Correspondence: Prof. Patrick M. Honore

Professor of Intensive Care Medicine, Director of the Critical Care Nephrology Platform, Intensive Care Department, University Hospital, Vrije Universiteit Brussel, Laarbeeklaan 101, B-1090 Brussels, Belgium.

E-mail: Patrick.Honore@uzbrussel.be

\begin{tabular}{|l|}
\hline Access this article online \\
\hline $\begin{array}{l}\text { Website: } \\
\text { www.intern-med.com }\end{array}$ \\
\hline DOI: \\
10.1515/jtim-2013-0003 \\
\hline Quick Response Code: \\
\hline \\
\\
\end{tabular}

\title{
Continuous renal replacement therapy allows higher colistin dosing without increasing toxicity
}

\author{
Patrick M. Honore ${ }^{1 *}$, Rita Jacobs ${ }^{1}$, Olivier Joannes-Boyau², Willem Boer ${ }^{3}$, Elisabeth \\ De Waele ${ }^{1}$, Viola Van Gorp ${ }^{1}$, Herbert D. Spapen ${ }^{1}$ \\ ${ }^{1}$ Intensive Care Medicine, Intensive Care Dept, Universitair Ziekenhuis Brussel, Vrije Universiteit Brussel, \\ Brussels, Belgium; ' ${ }^{2} \mathrm{CU}$ Consultant, Haut Leveque University Hospital of Bordeaux, University of Bordeaux \\ 2, Pessac, France; ${ }^{3}$ Department of Anaesthesiology and Critical Care Medicine, Ziekenhuis Oost-Limburg, \\ Genk, Belgium
}

\section{ABSTRACT}

Polymyxins are "old" antimicrobials which were abandoned for almost 30 years because of significant renal and neurological toxicity. However, the alarming rise of multi-resistant Gramnegative bacterial infections worldwide has revived interest in these "forgotten" agents. Colistin (polymyxin E) is one of the main antibiotics of this class. It is most often administered as the pro-drug colistimethate sodium. Doses for treatment of systemic infections in adults range between 3 and 9 million IU per day. Colistin is increasingly used for treatment of pneumonia and bacteremia in critically ill patients. During their ICU stay, many of these subjects will need continuous renal replacement therapy (CRRT) because of acute kidney injury or an unstable hemodynamic condition. Based on recent pharmacological data and own experience, we postulate that patients undergoing CRRT may receive substantially higher doses of colistin (i.e., a high loading dose, followed by a maintenance dose up to 4.5 million IU tid). Treatment can be continued for a prolonged time period without increasing toxicity. CRRT counteracts colistin accumulation because the drug is continuously filtered and also significantly adsorbed in the bulk of the dialysis membrane. Implementing such "CRRT rescue" therapy does require the strict use of highly adsorptive dialysis membranes in association with citrate anticoagulation to increase membrane performance.

Key words: acute kidney injury, colistin, continuous, colistin CRRT dosing, colistin high dosing, dialysis, nephrotoxicity, renal replacement therapy

\section{INTRODUCTION}

After being abandoned in the 1980 s because of a high incidence of nephro- and neurotoxicity, the polymyxin antibiotics have been recalled into service as a lastresort treatment of recently emerging multidrug-resistant Gram-negative infections. ${ }^{[1]}$ Colistin (polymyxin E) in particular is predominantly and increasingly used to treat nosocomial pneumonia and bacteremia caused by Acinetobacter baumannii, Pseudomonas aeruginosa, and Klebsiella pneumoniae .

Pharmacokinetic (PK) and pharmacodynamic (PD) characteristics of colistin are poorly documented. Colistin is mostly administered as the pro-drug colistimethate sodium which undergoes spontaneous hydrolysis to form active colistin. ${ }^{[2]}$ Doses used for systemic infections in adults range between 3 and 9 million IU per day. However, recent PK studies indicate that maximal colistin concentrations achieved following administration of currently recommended doses are low. ${ }^{[3]}$ Combination antimicrobial therapy (e.g., with a carbapenem) is proposed to maximize antimicrobial efficacy, but this approach has not proven clinical advantage over monotherapy. ${ }^{[4]}$ Higher colistin dosing is another option but at the cost of an increased risk for acute kidney injury (AKI). Either a high daily dose $\mathrm{e}^{[5]}$ or the total cumulative dose ${ }^{[6]}$ of colistin are considered to be potentially predictive for AKI. 
CRRT has become a standard adjuvant therapy in an ICU setting. Apart from commonly accepted indications (AKI of different origin, hemodynamic instability without AKI, fluid overload...), CRRT could also be used as a "supporting" therapy to allow adequate dosing of an antibiotic whilst limiting some or all of its known toxicities. Regarding colistin treatment, CRRT would thus be an elegant method to reconcile optimal efficacy and safety issues when high doses are used for an extended period of time.

\section{COLISTIN TOXICITY}

Detecting colistin toxicity at the bedside is cumbersome, in particular when patients are mechanically ventilated. Colistin neurotoxicity should be suspected in case of seizures, prolonged coma, or when a previously conscious patient suddenly fails to trigger the ventilator or develops unexplained respiratory muscle paralysis. ${ }^{[1,7,8]}$ A more commonly observed adverse effect following colistin administration is the development of nephrotoxicity. Risk factors for this complication include older age, pre-existing renal dysfunction, hypoalbuminemia, and concomitant use of intrinsically nephrotoxic drugs (e.g., non-steroidal anti-inflammatory agents, aminoglycosides, vancomycin). Measuring colistin serum levels is difficult, labor-intensive and only feasible in highly specialized laboratories. Moreover, any relationship between a distinct serum concentration and the occurrence of toxicity has not been demonstrated.

\section{COLISTIN HANDLING DURING CRRT AND ITS RELATION TO EFFICACY AND TOXICITY}

Colistin is predominantly non-renally cleared in patients with normal kidney function. ${ }^{[9]}$ Colistin undergoes extensive (up to $80 \%$ ) tubular reabsorption. As a consequence, most of the filtered colistin is retained in the body and only an extremely low fraction is excreted unchanged in the urine. It can be predicted from recent PK models that a high loading dose is paramount to obtain a rapid bacterial killing effect. Without such loading dose, steady-state bactericidal colistin concentrations are only reached after 24 to 48 h. ${ }^{[10]}$

Very few data exist regarding colistin treatment under conditions of renal replacement. Elimination of colistin has been assessed during intermittent ${ }^{[1]}$ as well as continuous $^{[12-14]}$ dialysis. However, these studies do not provide clinically relevant PK/PD information because colistin doses were either in a toxic ${ }^{[11]}$ or a rather low ${ }^{[12-14]}$ range. Colistin handling is dramatically altered during convective CRRT. In fact, no carrier- mediated mechanism returning colistin from the ultra filtrate to the blood is present which virtually excludes reabsorption. ${ }^{[9]}$ Also, the AUC:MIC ratio has been identified as the PK/PD parameter best associated with colistin efficacy. ${ }^{[7]}$ To maximize this ratio, higher doses of colistin may be warranted in the ICU setting. For these reasons, a substantially higher maintenance dose than in patients without AKI (4.5 million IU tid instead of bid) should be given during CRRT. ${ }^{[16,17]}$ Toxicity risk depends upon either drug accumulation by reabsorption or the presence of too high peak concentrations. During CRRT, continuous loss by filtration permits to avoid dose accumulation of colistin whilst bulk membrane adsorption avoids occurrence of huge or persisting peak concentrations. ${ }^{[9,16]}$ This explains the absence of colistin toxicity during CRRT even when the aforementioned high doses are administered for a long time period. Still, caution is needed since not all currently used membranes have similar adsorptive capacity. ${ }^{[18]}$

\section{CRRT-RELATED STRATEGIES TO AVOID COLISTIN TOXICITY}

\section{Dose and modality}

Continuous venovenous hemofiltration (CVVH), based on convective elimination under regional iso-natremic citrate anticoagulation, is the preferred CRRT modality in ICU patients. Continuous venovenous hemodiafiltration has not shown any superiority above CVVH for eliminating small molecules. ${ }^{[19]}$ The current KDIGO guidelines recommend a standard dialysis dose of $35 \mathrm{ml} / \mathrm{kg} / \mathrm{h}$ to ascertain delivery of at least $25 \mathrm{ml} / \mathrm{kg} / \mathrm{h} .{ }^{[20]}$ High-volume CVVH does not offer any more benefit. ${ }^{[21,22]}$

\section{Type of membrane}

Membranes with no or poor adsorptive capacity are not suitable for antibiotic clearance. Drug elimination in CRRT devices equipped with this type of membranes is entirely determined by convection. ${ }^{[12,13]}$ In contrast, the novel AN69 ST (Surface Treated) membrane allows much higher clearance through adsorption. Elimination occurs in part at (the rapidly saturated) membrane surface but predominantly in its (less easily saturated) bulk. ${ }^{[18]}$ Early saturation is unlikely and frequent membrane changes (e.g., every 12 to $24 \mathrm{~h}$ ) are not necessary. ${ }^{[16,18]}$ This is corroborated by our experience in a small cohort of patients who supported colistin doses as high as 4.5 million IU tid for more than five consecutive days without exhibiting any side-effects or toxicity while the membrane was not changed during this time period. ${ }^{[7,16]}$ Polymethylmetacrylate membranes may be capable to adsorb similar amounts of colistin into their bulk ${ }^{[18]}$ but studies reporting on colistin elimination using these particular membranes are lacking. Thus, high colistin doses can only be safely applied when 
highly adsorptive filters (such as the AN69 ST membrane) are employed. Monitoring of transmembrane pressure and hemopermeability index permit continuous assessment of functional capacity of the dialysis membrane. The absence of clotting is an indirect marker of absence of membrane clogging. ${ }^{[23]}$

\section{Anticoagulant therapy}

Citrate is the anticoagulant of choice because it effectively combats the clogging process thereby guaranteeing longterm optimal porosity ${ }^{[2]}$ and - when using the AN69 ST filter - adsorptive capacity of the membrane. ${ }^{[16]}$

\section{CONCLUSIONS}

Colistin is an intrinsically nephrotoxic and potentially neurotoxic antibiotic. Therapeutic drug monitoring for steering its administration is not available in daily ICU practice. Despite these "drawbacks," patients undergoing CVVH can support long-term colistin therapy at doses up to 4.5 million IU tid without toxicity. Implementation of such treatment protocol strictly requires the use of filters allowing high colistin adsorption in the bulk of the membrane in association with citrate anticoagulation for prevention of early membrane clogging in order to maintain high convection elimination as well.

\section{REFERENCES}

1. Spapen HD, Jacobs R, Van Gorp V, Troubleyn J, Honoré PM. Renal and neurological side effects of colistin in critically ill patients. Ann Intensive Care 2011;1:14.

2. Bergen PJ, Li J, Rayner CR, Nation RL. Colistin methanesulfonate is an inactive prodrug of colistin against Pseudomonas aeruginosa. Antimicrob Agents Chemother 2006;50:1953-8.

3. Couet W, Grégoire N, Marchand S, Mimoz O. Colistin pharmacokinetics: The fog is lifting. Clin Microbiol Infect 2012;18:30-9.

4. Yahav D, Farbman L, Leibovici L, Paul M. Colistin: New lessons on an old antibiotic. Clin Microbiol Infect 2012;18:18-29.

5. Deryke CA, Crawford AJ, Uddin N, Wallace MR. Colistin dosing and nephrotoxicity in a large community teaching hospital. Antimicrob Agents Chemother 2010;54:4503-5.

6. Hartzell JD, Neff R, Ake J, Howard R, Olson S, Paolino K, et al. Nephrotoxicity associated with intravenous colistin (colistimethate sodium) treatment at a tertiary care medical center. Clin Infect Dis 2009;48:1724-8.

7. Michalopoulos AS, Falagas ME. Colistin: Recent data on pharmacodynamics properties and clinical efficacy in critically ill patients. Ann Intensive Care 2011;1:30.

8. Spapen HD, Honore PM, Gregoire N, Gobin P, de Regt J, Martens GA, et al. Convulsions and apnoea in a patient infected with New Delhi metallo- $\beta$-lactamase-1 Escherichia coli treated with colistin. J Infect 2011;63:468-70.

9. Li J, Nation RL, Turnidge JD, Milne RW, Coulthard K, Rayner CR, et al. Colistin: The re-emerging antibiotic for multidrug-resistant Gram-negative bacterial infections. Lancet Infect Dis 2006;6:589-601.
10. Mohamed AF, Karaiskos I, Plachouras D, Karvanen M, Pontikis K, Jansson B, et al. Application of a loading dose of colistin methanesulfonate in critically ill patients: Population pharmacokinetics, protein binding, and prediction of bacterial kill. Antimicrob Agents Chemother 2012;56:4241-9.

11. Marchand S, Frat JP, Petitpas F, Lemaître F, Gobin P, Robert R, et al. Removal of colistin during intermittent haemodialysis in two critically ill patients. J Antimicrob Chemother 2010;65:1836-7.

12. Markou N, Fousteri M, Markantonis SL, Zidianakis B, Hroni D, Boutzouka E, et al. Colistin pharmacokinetics in intensive care unit patients on continuous venovenous haemodiafiltration: An observational study. J Antimicrob Chemother 2012;67:2459-62.

13. Cho YS, Yim H, Yang HT, Hur J, Chun W, Kim JH, et al. Use of parenteral colistin for the treatment of multiresistant Gram-negative organisms in major burn patients in South Korea. Infection 2012;40:27-33.

14. Li J, Rayner CR, Nation RL, Deans R, Boots R, Widdecombe $\mathrm{N}$, et al. Pharmacokinetics of colistin methanesulfonate and colistin in a critically ill patient receiving continuous venovenous hemodiafiltration. Antimicrob Agents Chemother 2005;49:4814-5.

15. Plachouras D, Karvanen M, Friberg LE, Papadomichelakis E, Antoniadou A, Tsangaris I, et al. Population pharmacokinetic analysis of colistin methanesulfonate and colistin after intravenous administration in critically ill patients with infections caused by gram-negative bacteria. Antimicrob Agents Chemother 2009;53:3430-6.

16. Honore PM, Jacobs R, Lochy S, De Waele E, Van Gorp V, De Regt J, et al. Acute respiratory muscle weakness and apnea in a critically ill patient induced by colistin neurotoxicity: Key potential role of hemoadsorption elimination during continuous venovenous hemofiltration. Int J Nephrol Renovasc Dis 2013;6:107-11.

17. Lee J, Han S, Jeon S, Hong T, Song W, Woo H, et al. Population pharmacokinetic analysis of colistin in burn patients. Antimicrob Agents Chemother 2013;57:2141-6.

18. Honore PM, Jacobs R, Joannes-Boyau O, De Regt J, De Waele E, van Gorp V, et al. Newly designed CRRT membranes for sepsis and SIRS-a pragmatic approach for bedside intensivists summarizing the more recent advances: A systematic structured review. ASAIO J 2013;59:99-106.

19. Morimatsu H, Uchino S, Bellomo R, Ronco C. Continuous renal replacement therapy: Does technique influence electrolyte and bicarbonate control? Int J Artif Organs 2003;4:289-96.

20. Joannes-Boyau O, Honoré PM, Perez P, Bagshaw SM, Grand H, Canivet $\mathrm{JL}$, et al. High-volume versus standard-volume haemofiltration for septic shock patients with acute kidney injury (IVOIRE study): A multicentre randomized controlled trial. Intensive Care Med 2013;39:1535-46.

21. Zhang P, Yang Y, Lv R, Zhang Y, Xie W, Chen J, et al. Effect of the intensity of continuous renal replacement therapy in patients with sepsis and acute kidney injury: A single-center randomized clinical trial. Nephrol Dial Transplant 2012;27:967-73.

22. Kellum JA, Lameire N, For the KDIGO AKI Guideline Work Group. Diagnosis, evaluation, and management of acute kidney injury: A KDIGO summary (Part 1). Crit Care 2013;17: R204.

23. Honore PM, Joannes-Boyau O, Collin V, Boer W, Gressens B, Janvier G. Practical daily management of extra-renal continuous removal. Reanimation 2008;17:472-8.

24. Hofbauer R, Moser D, Frass M, Oberbauer R, Kaye AD, Wagner O, et al. Effect of anticoagulation on blood membrane interactions during hemodialysis. Kidney Int 1999;56:1578-83.

How to cite this article: Honore PM, Jacobs R, Joannes-Boyau O, Boer W, De Waele E, Van Gorp V, et al. Continuous renal replacement therapy allows higher colistin dosing without increasing toxicity. J Transl Intern Med 2013; 1(1): 6-8.

Source of Support: Nil, Conflict of Interest: None declared 